\title{
The Harmony of the Physical Vacuum
}

\author{
Anatolii Pavlenko* \\ Open International University of Human Development "Ukraine", LLS "Spinor \\ International", Kyiv, Ukraine \\ *Corresponding Author: Anatolii Pavlenko, Open International University of \\ Human Development “Ukraine”, LLS “Spinor International”, Kyiv, Ukraine.
}

Received: February 26, 2020

Published: April 09, 2020

(C) All rights are reserved by Anatolii Pavlenko.

\begin{abstract}
This paper is a summary of ideas and methods related to protection of people from the negative impact of electronic technology. While conducting research, the author intended to clearly define the prevailing negative impact of torsion fields in comparison with electromagnetic radiation. Electromagnetic fields warm the tissues of a person using all sorts of gadgets, while torsion fields provide influence at the gene level. Much attention is paid to the technology of protection from torsion fields. For a more complete understanding of the problem of protecting the population from the negative influence of torsion fields, the authors considered the concept of the "fabric of the universe" developed by several authors, which enabled representing the processes occurring in Physical Vacuum in a more distinct way. The fact that virtual particles have their own subconscious nature, which differs from ours that was predicted by N. Tesla, has been confirmed.

Keywords: Physical Vacuum; Torsion Fields; Harmonization of Physical Vacuum; Subconscious Feature of Virtual Particles
\end{abstract}

\section{Introduction}

We face the task of breaking through the fog of unprepared human consciousness and have started to define our ability to defend ourselves from the negative impact of electronic technology.

What I propose is a completely different direction in protecting people from the negative impact of electronic equipment and it enables to create conditions to resolve the environmental and energy problems that the mankind faces today. In essence, the paper deals with the technologies for protecting the gene pool of people exposed to torsion radiation, and not to electromagnetic radiation.

We must solve the problem of harmonizing the Physical Vacuum, which is an integral part of the environment.

Harmony is a complex semantic concept that has several layers.

In the history of the teachings on harmony, the most diverse types of understanding were applied. The word "harmony" also denotes the order of nature and the cosmos, the beauty of the physical and moral world of man, etc. Harmony was understood as the equality or proportionality of parts with each other or of the whole. Such understanding was applied, as a rule, in a certain mathematical form and was expressed in the form of numerical proportions. This is the most external, formal and empirical understanding of harmony. It draws attention to the quantitative side of the matter and is indifferent to the original qualitative features of the parts entering into harmonic correspondence. The mathematical understanding of harmony captures primarily the quantitative determinism of harmony.

However, none of these layers of harmony can be applied to the Physical Vacuum while it is in an unexcited state. Virtual particles of the Physical Vacuum move in different directions, disappearing and appearing in this movement (Figure 1).

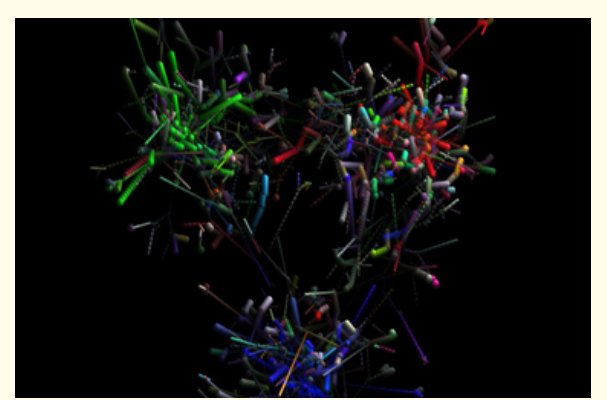

Figure 1: Physical vacuum fluctuations.

\section{Physical vacuum}

Until recently, direct experimental observation of this fundamental phenomenon was considered impossible. Scientists proceeded from the fact that vacuum fluctuations always manifest themselves in nature indirectly, leading to a wide range of effects.

Physicists from the laboratory of Professor Alfred Leitenstorfer [1] found vacuum fluctuations, and access to the ground state of the quantum system was obtained without any increase of its intensity. Until recently, direct experimental observation of this fundamental phenomenon was not considered possible. They demonstrated the first direct observation of the so-called Physical Vacuum fluctuations by using short light pulses while employing highly precise optical measurement techniques.

The "virtual chain" of transformations of the Physical Vacuum is described in [2] (http://darkenergy.narod.ru/mhpicru.html). A virtual photon, after passing a certain distance, becomes a virtual electron-positron pair. The electron and positron make a half - turn in opposite directions, drawing a circle in space. At the junction they disappear and generate a virtual photon, which moves further. 
It was pointed out that in quantum field theory vacuum fluctuations are interpreted as the creation and destruction of virtual particles (that is the particles that are continuously generated and immediately destroyed), or virtual quanta of a given field [3]. In a vacuum, different pairs of particle-antiparticles are produced, and these pairs can be based on electrons, protons, neutrons, quarks, etc. Virtual particles participate in the same way in interactions, as real ones. A virtual photon is capable of generating a virtual electron-positron pair, analogous to the production of a real photon of a real electron-positron pair.

It is obvious that the description of the virtual particles state in Physical vacuum in non-excited state is very difficult, since virtual particles have a very short lifetime and they undergo transformations. It is known that anything emerging in this world, including living beings, polarizes the Physical Vacuum. The picture of Physical Vacuum polarization is shown on figure 2.

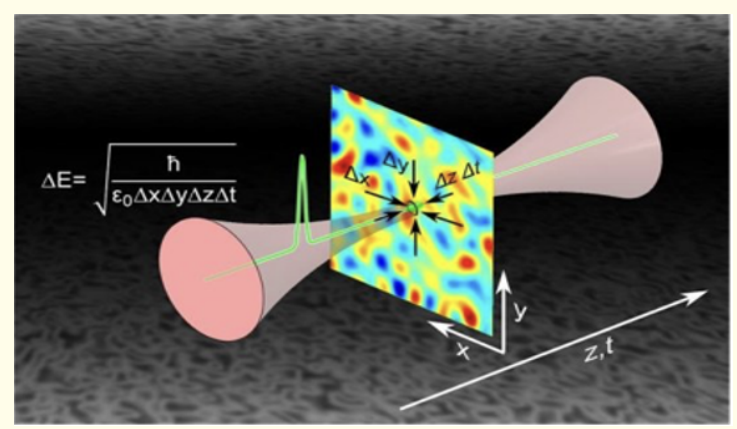

Figure 2: Polarization of physical vacuum.

This polarization of the Physical Vacuum (PV) is created for example by TV-sets, computer monitors, and other electronic gadgets. The action produces a charge polarization of the vacuum, as prescribed by quantum electrodynamics. The virtual particles, so rearranged, interact differently with the longer light wave. In built up pockets they make the light wave noisier while in the depleted regions the light wave is quieter. For the purpose of general understanding of this phenomena essence, it is necessary to address some theoretical questions related to interaction of the real and virtual particles. The polarization model of the PV associated with the perturbation of virtual electrons by a real electron $\mathrm{E}$ appeared in 2007 in a book by Roger Penrose [4]. One can become familiar with the process of polarization in the interaction of a real electron with the PV in [4].

Here it is necessary to quote the words of M. Gell-Mann: "Quantum mechanics is a discipline of complete mystery and paradoxes, which we do not understand, but we can use". Quantum entanglement is a fundamental phenomenon that reveals deep connections between quantum mechanics and phonon-photon entanglement and it has practical applications as a key resource in quantum information processing. It remains challenging albeit promising for both fundamental physics and technological applications.

Undoubtedly, the unity of the world is of information nature. The particle's strategy - the wave function- is in the consciousness of the particle and is the result of the work of this consciousness related to the information about the world.

Thus, the particle solves the quantum-mechanical problem. When new information is received, the particle adjusts its strategy i.e. it corrects its wave function. A striking example of such correction is the fact that if you identify nodes of left and right geopathic zones in a closed space, for example, in an apartment, and connect these nodes themselves with a conductive wire or a non-conductive rope, then space harmonization takes place in accordance with AE Akimov's model. In this case, virtual electrons and positrons are embedded in each other, forming a "phyton" in accordance with $\mathrm{AE}$ Akimov's mode [3].

In other words, control is understood as an action in which the impact on the PV, aimed at harmonization, has an indirect informational effect.

The information influence is provided by the device which has two zones where the right and left torsion fields are registered, and these areas are connected. In this case, virtual particles get the command to be embedded or penetrate into each other. The PV consisting of virtual particles is oriented toward this information influence. Virtual electrons and positrons go into the mode of "phytons" when these virtual particles are put together, i.e. components of the PV are placed in symmetry mode. In this case, there is no negative impact on the environment and people. It is understood that self-organization is carried out using the subconscious feature of virtual particles.

Many people have doubts about this approach, but long time ago Nikola Tesla determined subconscious characteristics of real particles.

Tesla was convinced that everything in the Universe, down to the electron (at that time there was not a conception about virtual particles) has consciousness. The universe is a whole organism, which consists of many parts, but differing in the frequencies of vibrations. Each part is a parallel world. Having entered resonance with the frequency of another world, we open a window into this parallel world. Space is a single intelligent living organism.

Tesla treated electricity as a living creature. He could talk to it and give it his orders. Each elementary particle - electron, positron, proton and other - is a complex organized entity. It is a living intelligent being, in its spiritual level, it is not simpler than you and me, and it's not unusual to agree with them and get in touch with them. I do not adhere to such views alone. There are a lot of people and theorists, and basically - practitioners who face such things and are reconsidering their worldview".

Today it is established that elementary particles can obey the mental effects and directions of a person, and this does not fit into the known physical laws, but is proved in a variety of experiments. The experiments were conducted in which the laser beam deflected the thought action, the water changed its properties at a distance of thousands of kilometres, the plane of polarization of the light rays 
changed, etc. The phenomenon was called "weak mental impact". People who are far away from the laboratory act mentally on how electrons behave when they pass through a diffraction grating, etc. All this is documented and completely beyond any theoretical description.

Tesla wrote that the main purpose of his life was to point out phenomena and disseminate ideas, which can be the starting points for new research.

There is no person since Tesla's death who could successfully provide a real description of processes in a PV, taking into account the subconscious feature of elementary particles.

Feynman indicated: "I think I can responsibly declare that no one understands quantum mechanics. If possible, stop asking yourself how this is possible? - because it will lead you to a dead end, from which no one has to get out yet". It's clear why it is difficult to understand a quantum mechanics because the quantum mechanics is constructed purely phenomenologically, that is, quantum mechanics is simply a physical model of experimentally observed quantum phenomena, which reflects these phenomena, but does not reveal their fundamental physical meanings.

The picture being drawn by quantum mechanics is very difficult to comprehend the picture of the world. There is a problem of the consciousness of living and elementary particles, and many researchers assume the existence of a deep connection between these two secrets: why this connection exists, on what it is based and what its mechanism is. The only thing that can be said about this connection is that it exists. We will try to shed light on some quantum phenomena. Since the problem of consciousness is no less mysterious.

\section{Materials and Methods}

\section{Ways to harmonize physical vacuum}

Even if Nature is a colossal laboratory, and the Universe is a giant computer, all the same, they are based on a very limited number of algorithms-laws that will enable harmonizing PV. By the harmonization of $\mathrm{PV}$, we understand the elimination of the negative impact on humans of all electronic equipment, including the influence of geopathogenic zones of the Earth.

However, we begin with the history of the teachings on harmony, in which the most diverse types of its understanding have been put forward. The word "harmony" also meant the natural order of nature and the cosmos, the beauty of the physical and moral world of man, etc. Harmony is a complex semantic concept that has several layers. First of all, we can talk about a purely mathematical understanding of harmony, which is characteristic of the whole history of aesthetic thought. Harmony was understood as the equality or proportionality of parts with each other or parts as a whole. Such an understanding took, as a rule, a certain mathematical form and was expressed in the form of numerical proportions. This is the most external, formal and empirical understanding of harmony. It captures attention to the quality originality of parts entering into harmonic correspondence. Most recently, we started talking about the harmonization of PV in order to prevent its negative impact on the living beings.

According to a number of signs, the algorithms-laws are sometimes grouped in the most unexpected way, and their number is extremely small, as well as fundamental laws.

We list some of them: the harmonization of the PV using a mechanical connection of conductive or non-conductive material regions with virtual electrons and virtual positrons, for example, "minus" and "plus" nodes of geopathogenic zones of the Earth, a similar result reveals the connection of the poles of the magnet, and it is typical that in the daytime, the north magnetic pole has a right torsion field, and at night it has a left one. Magnesium, unlike other proven metals, has a right torsion field during the day. If we put a magnesium plate on the end of a vertically standing tube of small diameter, which generates a left torsion field from the end in the afternoon, then the PV harmony mode is established in an enclosed space. At night, everything will be the other way around, but the state of harmonization of the Physical vacuum is maintained. Similar phenomena are observed in wildlife: a cactus generates a right torsion field during the day, and a left one at night, similar to a magnet. There are many such examples.

At the verbal level, the harmonization of PV is realized by singing mantras, for example, the OUM mantra, or by playing only one "C" note. PV can be harmonized with the help of the sound of Tibetan and Indian bowls. The essence of Keeley's theory is based on the idea that the external musical tones of sound resonate with vibrations - the responses of the atom of the working fluid through the ether.

All the listed phenomena of harmonization, except harmonization at the verbal level, are related to the so-called "entangled state", i.e. in this case, we carry out mechanical "entanglement" by connecting elements with opposite signs with a simple rope or conductive material.

An interesting entanglement phenomenon can be accomplished with the help of a photograph of a geopathic zone node.

When photographing any objects with modern cameras, the torsion fields of the complex material medium entering the input of devices with charge coupling together with the electromagnetic (light) flux change the orientation of the spins of the atoms of the recorded information in such a way that the spins of the recorded information repeat the spatial structure of this external torsion field. As a result, in any photograph stored in the range of devices with charge always, in addition to the visible image, there is always an invisible torsion image.

If the printed photograph of the node of the geopathogenic zone of a certain room is put on the geopathogenic zone, then the space harmonization mode is set in this room.

Another option of harmonization can be achieved by connecting the cones in the left and right fields, respectively, depicted in 
figure 2. This action is similar to the harmonization of the Physical Vacuum in a confined space by combining the "minus" and "plus" nodes of the geopathogenic zones of the Earth.

R. Openheimer wrote: "We do not understand the nature of matter, the laws that govern it, and the language in which it should be described".

A gradual approach to the truth of cognition of the phenomena of the world around us is the real path, the criterion of which is the ability to predict certain phenomena and use them for the survival on this planet. It may take considerable time to comprehend the results of this article in order to combine the visual images of human thinking and other factors with the secrets of nature.

\section{More detailed}

Let's look at several hypotheses that state that processes in the Universe are based on the properties of fundamental numerical constants - quantities whose values do not change. Mathematical constants are defined independently of any physical measurements. It is believed that their true origin is not completely clear, although in most cases they are interpreted quite fully.

It is known that mathematics operates with a set of abstract form-structures and does not belong to the natural sciences; it is widely used to accurately formulate their contents and obtain new results.

If we talk about understanding harmony from the point of view of $\mathrm{PV}$, then harmony is represented as the unity of equal parts with opposite characteristics, i.e. symmetric embedding of a virtual electron and positron. Such understanding can take a certain mathematical form and can be expressed in the form of numerical proportions.

There is a perception that people did not create mathematics; they discovered it. It is a universal language and law.

Many researchers believe that space consists of discrete parts, like much else in nature. This is explained in detail in the film: https://www.youtube.com/watch?v=jZChu45vfOg.

In this video, the author calls to recognize the equality of the electron and the positron, which will make it possible to explain many of the deadlock problems of modern physics.

A detailed examination of the fabric of the universe allows us to see the "rhombic" structure of the cells of space, and this is similar to the sections of ordinary packed cones.

The fabric of the universe was built on the basis of magic squares using numbers from 1 to 9 .

A sample image of the fabric of the universe is shown in figure 3.

The idea of a new discipline "mathematical principles (foundations) of harmony" belongs to the scientist and composer M. Marutaev, who was born in Donetsk (Ukraine). This theoretical rigorously terminological concept was first proposed to him in

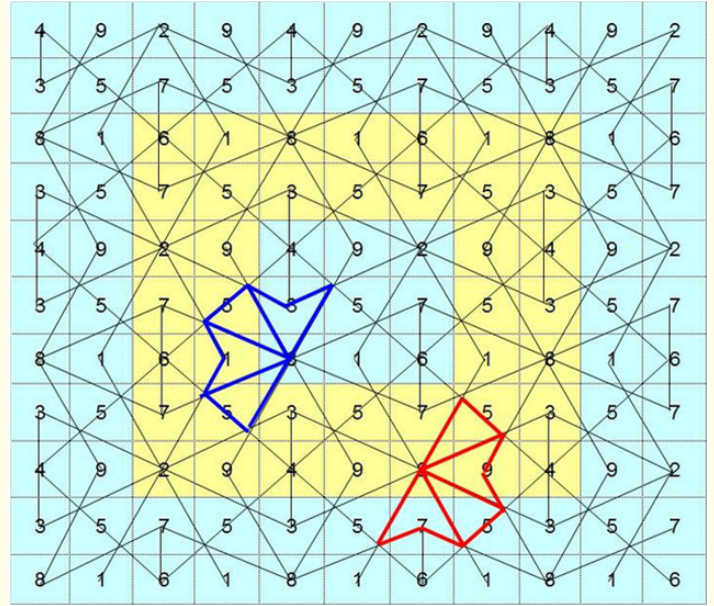

Figure 3

a collective monograph of 1990 [5] in the section "Harmony as a regularity of nature".

The natural scientific content of the concepts of "harmony", "golden section" and their manifestations in architecture, music, psychology of perception and shaping of wildlife, as well as in the periodic table, planetary distances, macrocosm and microcosm, etc. are studied. Many problems are posed and highlighted. First he expressed the hypothesis about the numerical harmony of the universe. According to the author, numerical harmony in the Universe is determined by three interrelated laws: qualitative symmetry, broken symmetry, golden ratio. Marutaev saw harmony in the form of a paradoxical identity of opposites on the basis of the axiomatic construction of the theory of harmony. All fundamental constants are explained through all possible sections of a regular cone (Figure 4). Here, the conical packing of the Euclidean space is used that provides maximum density, which is expressed in terms of the golden ratio.

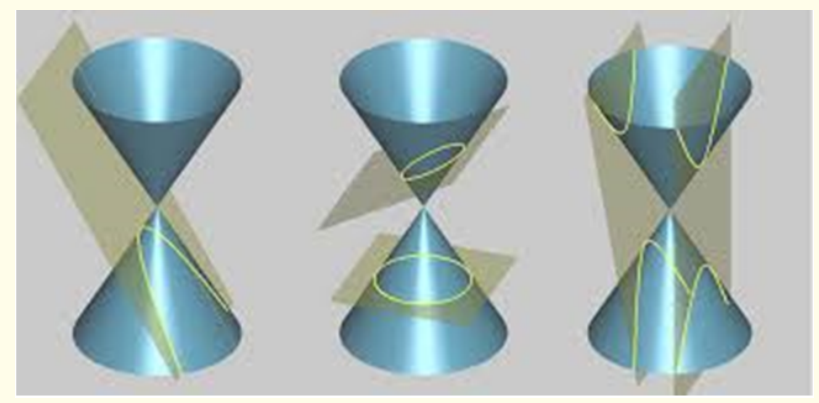

Figure 4

In connection with the incomprehensible nature of matter, the world scientific community, along with the classic implicit presentation of knowledge, has a different direction, different from purely mathematical methods. Fundamental science is still not able to explain neither the devices of levitation of D. Keeley, nor the suits of W. Schauberger and D. Searle and others. D. Keeley managed to build a bridge between visible and invisible matter and energy, and visible matter is only a product of invisible. 
When developing their original devices, Tesley and Keely used their intuitively-acquired thinking, which they acquired in the process of research, in which they used their body, eyes, ears and nervous system as an intelligent detector, with which no technical detectors can be compared.

In [6], "torsion mathematics" was considered to be discovered by of Mark Rodin, anticipatory, according to some authors, our time. Using 9 single-digit numbers, they use the style of these numbers in such a way that each number displays the number of angles in its style. For example, a unit has one corner; two units have two angles, etc. It is known that the face of any figure will have its own torsion field depending on the number of angles in the face of the figure. Mark Rodin discovered that the numbers are geometry. Numbers do not model - they are models. All "torsion mathematics" is based on the use of 9 single-digit numbers (Figure 5). During his research, Marco Rodin stated: "I devised and designed not only my own tools, but tools that are based on perfection, the axioms, the universal truths of the universe. Essentially, I claim to have discovered perfection." According to these studies, Marco's unique mathematical perspective provides detailed insight into the nature of the universe.

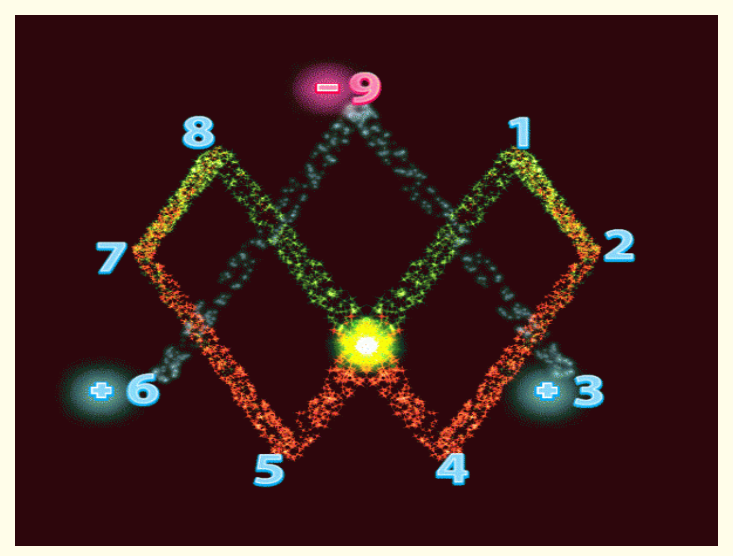

Figure 5

Nikola Tesla said: "If you only knew the magnificence of the numbers 3, 6 and 9, you would have owned the key to the universe".

More detailed ideas can be found in [6].

All that unites the mentioned variants of the fabric of the universe is that in all variants the fabric of the universe is visible in the form of a conical packing of space.

It is difficult to imagine how easily these approaches to the fabric of the universe can be combined; this is the task of the future. Scientists have to explain the mechanism of the causes of such interactions, as well as the structure of the source fields.

Perhaps, it is the harmony of the Physical Vacuum that can become the unifying beginning of the third millennium in the history of mankind, taking responsibility for adequately reflecting the challenges of time with a clear life-affirming goal.
As the only chance that is still able to divert humanity from all the negative aspects that have been accumulated and acquired during civilization period. Here it is necessary to quote the words of M. Gell-Mann: "Quantum mechanics is a discipline of complete mystery and paradoxes, which we do not understand, but we can use". We will consider the Physical vacuum as a system of ring wave packets of electrons and positrons, but not as actual electron-positron pairs (Figure 1) [7].

We emphasize that with such approach we obtain a convenient, although different from modern concepts a simple model of the Physical Vacuum, which enables explaining many phenomena inexplicable based on the traditional approach.

Within the reasonable assumptions, it is not difficult to see that the condition of the true neutrality of the electron-positron Vacuum will be the state, in which the ring rotation packets of the electron and the positron are nested inside each other. The spins of these nested ring packets are opposite, then such a system will be self-compensating not only for charges, but also for classical spin and magnetic moment. In this case, there will be no negative impact on the living organisms. We will outline several variants to Harmonize Physical Vacuum by different methods, some of which at this stage could not be explained.

Let us consider an issue of information management. Information management is considered as an action where the controlling influence on the Physical vacuum with the aim of its harmonizing has an implicit, indirect information influence. The information impact is provided by the subject, which has two regions where the right and left torsion fields are recorded, and these areas are connected together. In such a case, virtual particles receive the command to become embedded or enter one into another. A physical vacuum composed of virtual particles is oriented toward this information impact direction. Virtual electrons and positrons move into the mode of "phytons" [7], when these virtual particles are merged, i.e. the components of the Physical Vacuum are in a symmetric mode. In this case, there is no negative impact on the environment and humans. It is understood that self-organization is arranged using the subconscious feature of the virtual particles themselves.

Many people can doubt this approach, but subconscious feature of virtual particles was described also by Nikola Tesla in his time.

With respect to a number of experiments, it became possible to put aside the phenomenological description and approach interpretations at the level of the process. In particular, it has become possible to determine in general a new approach to the interpretation of the experiments already performed and which lead to understanding of consciousness of the elementary particles.

\section{Colour}

Employees of SpinorLab under the supervision of the researcher Dr. Alexander Zarichanskyi discovered several options for harmonization of the Physical Vacuum by finding a combination of objects with different colours, no matter whether it is a sheet of 
coloured paper or, for example, combination of the respective colours of plasticine [7]. Leaving aside the model representation and physical reasoning of the discovered ways to eliminate the Earth geopathic zones at residential and industrial buildings, we present the list of determined combinations of energy-free blocking colours: 1 . a black sheet with an orange or light blue edge; 2 . a yellow sheet with a brown edge; 3 . an orange sheet with a yellow edge; 4 . a green sheet with a purple edge. 5 . a red sheet with a green edge; 7 . a blue sheet with a white edge; 8 . a purple sheet with a red edge; 9 . a white sheet with a green edge; 10 . a light blue sheet with a white edge or with a yellow edge.

Emission of the blue-colour lamp blocks geopathic zones of the Earth within indoor spaces, regardless of whether it is an incandescent lamp or a gas discharge lamp. It was found out that all combinations of colours, which block negative effects of geopathic zones on humans, can provide reliable protection from the adverse effects of mobile phones. Small-size combinations of coloured sheets may be placed on a mobile phone, laptop and other gadget battery. These same figures block generation of a spin pendulum. A twisted-pair wire of Internet generates the left torsion field. If there is a sheet of paper with numbers 17 (00010001) or 33 (01000001) written on it placed on this twisted pair, the left torsion field will be absent. By placing a coloured stripe (edge) on the base colour (background) we get a torsion-active system.

\section{Additional material}

We will proceed from the assumption that physical processes in the Universe are based on the properties of elementary particles, including the sub-consciousness of virtual particles. With their help, if not all, then the vast majority of occurring events are described. And the most amazing thing is that when you place a piece of paper in the geopathogenic zone with a written, for example, Euler formula, connecting the complex exponent with trigonometric functions for any real number $x$ : eix $=\cos x+i \sin x$, where e is the base of the natural logarithm, $\mathrm{i}$ - imaginary unit, comes the harmonization of PV in a confined space. All facts of the establishment of harmonization are confirmed by measurements with a VEGA-12 type instrument. This is another confirmation of the presence of the sub-consciousness in virtual particles. It is generally believed that the true origin of the subconscious feature of virtual particles is not completely clear, but numerous facts confirm its existence.

I believe that from a scientific point of view it is legitimate to raise the question of whether science, and in particular, the physics of torsion fields and biology, are too far from the entire spectrum of possible measuring devices (including those, for which we currently have no adequate interfaces, except language and discourse between people), which may be required for the correct measurement of some interesting phenomena.

\section{Results and Discussion}

1. A large number of options for harmonizing the PV are experimentally confirmed, which guarantee the minimizing the impact of electronic equipment on the living beings. By the harmonization of $\mathrm{PV}$, we understand the elimination of the negative impact on human beings of all electronic equipment, including the influence of geopathogenic zones of the Earth.

2. Harmonization of the PV can be carried out both by mechanical connection of space nodes with opposite signs of the torsion field with conductive or non-conductive material. The simplest confirmation of this thesis is the effect of combining the left and right nodes of the geopathogenic zones of the Earth, in which there is no negative effect of torsion fields on living beings.

3. PV can be harmonized by highlighting the enclosed space in blue.

4. PV can be harmonized on a verbal level by singing the OUMA mantra or songs with a large number of SI notes.

5. When placing an artificial structure into a confined space with a pre-recorded left and right field connected together, the PV of this room is harmonized.

6. Placing a geopathic photograph in some room on this same geopathic zone leads to harmonization of the PV of the premise.

7. A similar result is obtained by connecting the poles of the magnet with any material, and it is characteristic that the north magnetic pole has the right torsion field in the daytime and the left in the night. At night, everything will be the other way around, but the state of harmonization of the Physical vacuum is maintained.

8. Magnesium, unlike other proven metals, has a right torsion field during the day. If we put a magnesium plate on the end of a vertically standing tube of small diameter, which generates a left torsion field from the end in the afternoon, then the PV harmony mode is established in an enclosed space.

9. The negative influence of the torsion fields of the base station of mobile phones can be eliminated by placing an artificial structure near the station with pre-recorded left and right fields connected together.

10. It is possible to eliminate the negative influence of the torsion fields of the base station of mobile phones remotely by placing an artificial structure on the photograph of the base station. This could be a device like "Vernada-Odo". It is well known that the Base Station and its photograph are in a confused state and placing on the photograph of the base station an artificial structure consisting of a pre-recorded left and right field on the corresponding host, connected together, ensures the elimination of the negative impact of the station on the environment.

11. Many other options for eliminating the negative effects of electronics on living beings will be presented in future publications. 


\section{Conclusion}

A large number of options for harmonizing the PV are experimentally confirmed, which guarantee the impact minimizing of electronic equipment on the living beings.

It is simply surprising that the laws of harmonization are inevitable-that there is some inevitability of harmonization of PV, which can be carried out using a small number of actions, which then lead to harmonization.

\section{Bibliography}

1. Moskalenko AS., et al. "Direct sampling of electric-field vacuum fluctuations". Science 350.6259 (2015): 420-423.

2. http://darkenergy.narod.ru/mhpicru.html

3. Anatolii Pavlenko. "Possible ways to neutralize biopathogenic radiation". International Journal of Current Research 9.7 (2017): 53790-53799.

4. Roger Penrose. "The Road to Reality. A Complete Guide the Laws that of the Universe". London, Jonathan Cape (2004): 912.

5. Shevelev I Sh., et al. "Golden ratio: Three views on the nature of harmony”. M: Stroyizdat (1990): 343.

6. https://www.youtube.com/watch?v=mF_DcNQV_TU

7. Pavlenko A. "Biosafe electronics. Step beyond the horizon of official science”. Lambert Academic Publishing (2014): 226.

\section{Assets from publication with us}

- Prompt Acknowledgement after receiving the article

- Thorough Double blinded peer review

- Rapid Publication

- Issue of Publication Certificate

- High visibility of your Published work

Website: www.actascientific.com/

Submit Article: www.actascientific.com/submission.php Email us: editor@actascientific.com

Contact us: +919182824667 